\title{
On the determination of the dissipation rate of turbulence kinetic energy
}

\author{
James M. Lewis ${ }^{1}$ (D) Timothy W. Koster $^{1} \cdot$ John C. LaRue ${ }^{1}$
}

Received: 14 December 2020 / Revised: 20 May 2021 / Accepted: 12 June 2021 / Published online: 1 July 2021

(c) The Author(s) 2021

\begin{abstract}
The paper presents a comparison of the dissipation rate obtained from numerical differentiation of the time-resolved velocity, analog differentiation of the hot-wire signal, integration of the velocity derivative spectra obtained from the velocity spectra, and the application of a power decay law. Hot-wire measurements downstream of an active-grid provide the timeresolved velocity with a Taylor Reynolds number in the range of 200-470, turbulence intensities in the range of 5.8-11\%, and nominal mean velocities of 4,6 , and $8 \mathrm{~m} \mathrm{~s}^{-1}$. The dissipation rate calculated using a ninth-order central-difference scheme differs at most by $\pm 4 \%$ from the value obtained by analog differentiation. For comparison, a 23rd-order central-difference scheme offers negligible $(0.02 \%)$ difference relative to the ninth-order scheme. Correction for an apparent uncertainty in the calibration of the analog differentiator reduces the difference to $\pm 2.5 \%$. In contrast, integration of the velocity derivative spectra obtained from the velocity spectra leads to a dissipation rate $14-45 \%$ larger than the corresponding values obtained using analog differentiation. Results obtained from the application of a power decay law of turbulence kinetic energy with a nonzero virtual origin to determine the dissipation rate deviate by $1.7 \%, 1.6 \%$, and $3.6 \%$ relative to the corresponding values obtained from the analog differentiator based on the ensemble average of downstream locations with a $\pm 5.6 \%$ scatter about the ensemble average.
\end{abstract}

James M. Lewis

lewisjm@uci.edu

1 Department of Mechanical and Aerospace Engineering,

University of California, Irvine, Irvine, CA 92697, USA 


\section{Graphic abstract}

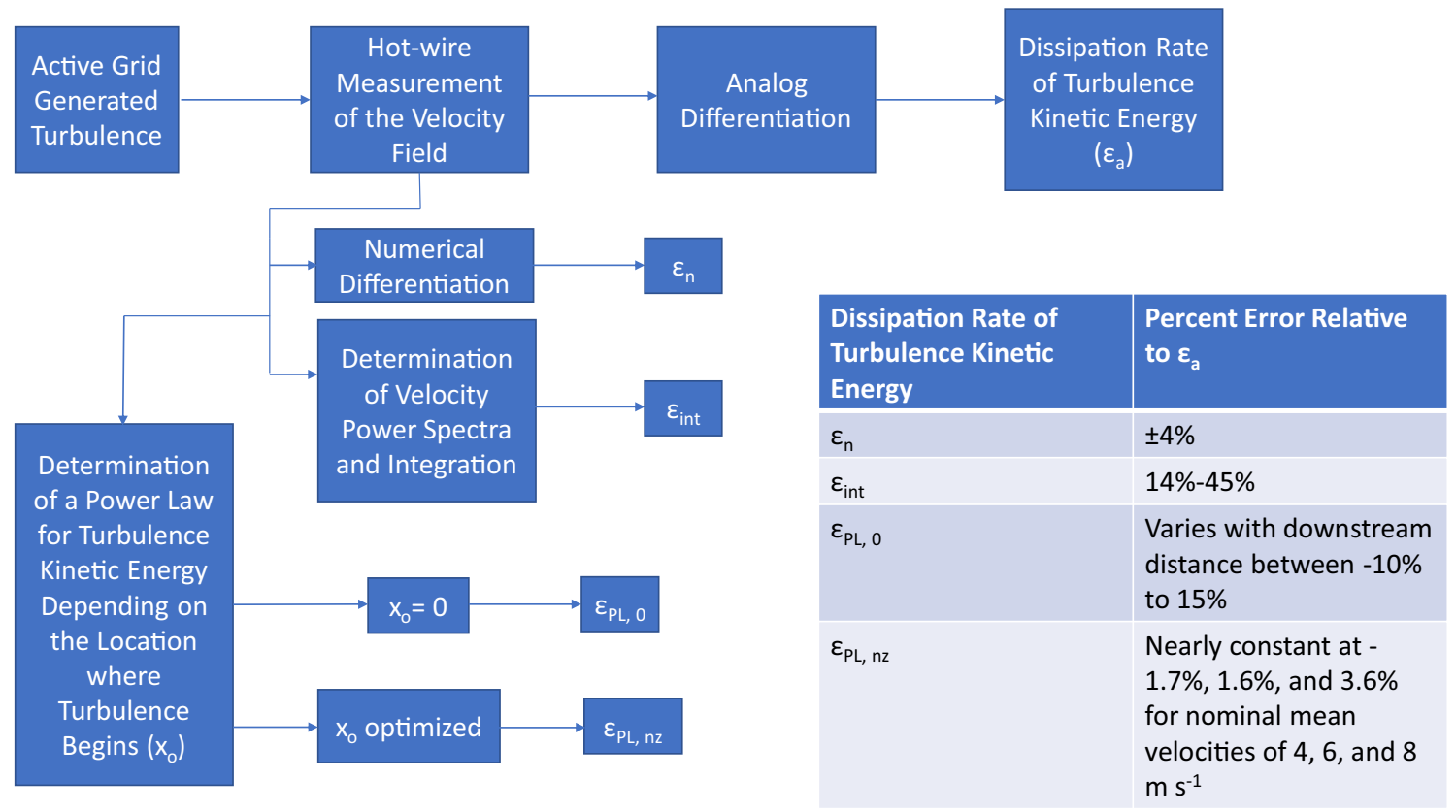

\section{Introduction}

The dissipation rate of turbulence kinetic energy $(\varepsilon)$ plays an important role in the study of turbulence. Different approaches exist to determine $\varepsilon$ from hot-wire data, with few comparisons made between the different methods. The study presented herein focuses on the relative accuracy of four approaches to determine the dissipation rate in nearly homogeneous isotropic turbulence. Two approaches rely upon the definition of the dissipation rate for homogenous and isotropic turbulence defined as:

$\varepsilon=15 v \overline{\left(\frac{\partial u}{\partial x}\right)^{2}}$

where $u=U-\bar{U}$ represents the fluctuating component of the downstream velocity $(U)$ at a downstream position, $x$. The dissipation rate also depends on the kinematic viscosity $(v)$. The notation $\left(^{-}\right)$indicates a time average of the quantity. Determining the quantity $\frac{\partial u}{\partial x}$ presents a significant challenge in experiments as its direct measure would require placing multiple sensors in the flow, which in turn influences the measurement. One approach to address this challenge relies on measuring the temporal derivative of the velocity $\left(\frac{\partial u}{\partial t}\right)$ and utilizing Taylor's hypothesis defined for low-intensity turbulence as: $\overline{\left(\frac{\partial u}{\partial x}\right)^{2}}=\frac{1}{\bar{U}^{2}} \overline{\left(\frac{\partial u}{\partial t}\right)^{2}}$

to relate the temporal to the spatial derivative. The application of Eq. 2 requires careful consideration of the speed at which the eddies propagate downstream as it may differ from the measured flow velocity (cf. del Álamo and Jiménez 2009).

The moderate-intensity turbulence observed in the turbulent flow downstream of an active grid-described in Sect. 2-requires a modified form of Taylor's hypothesis. Heskestad (1965) suggests a correction to Taylor's hypothesis for moderate intensity turbulence and Champagne (1978) applies a Taylor series expansion to Heskestad's correction to arrive at the relationship:

$\varepsilon=\frac{15 v}{\bar{U}^{2}} \overline{\left(\frac{\partial u}{\partial t}\right)^{2}}\left(1+\frac{\overline{u^{2}}}{\bar{U}^{2}}\left[1+\frac{2}{I_{u v}}+\frac{2}{I_{u w}}\right]\right)^{-1}$

using the isotropy ratios defined as $I_{u v}=\overline{v^{2}} / \overline{u^{2}}$ and $I_{u w}=\overline{w^{2}} / \overline{u^{2}} . v$ and $w$ represent the two cross-stream components of the fluctuating velocity oriented $90^{\circ}$ apart in the $y$ and $z$ directions, respectively.

The first approach to determine the temporal derivative of the velocity utilizes an analog differentiator, 
which directly differentiates the hot-wire voltage signal. A second approach uses numerical differentiation of the time-resolved velocity to determine the temporal velocity derivative (cf. Hearst et al. 2012). Hearst et al. analyze the effect of various orders of finite central-difference schemes on the corresponding dissipation rate. The authors conclude that higher order central-difference schemes result in the most accurate prediction of the dissipation rate due to the higher order central-difference schemes providing less attenuation of the power spectral values at high wavenumbers compared to the lower order central-difference schemes. Hearst et al. recommend the use of a 7-point or 9-point central-difference scheme based on balancing accuracy with noise amplification. The study herein quantitatively assesses the impact of order on the determination of the dissipation rate.

A third approach determines the variance of the temporal velocity derivative and the dissipation rate from the velocity power spectra, noted by Antonia (2003) and Mydlarski and Warhaft (1996), as:

$\varepsilon=15 v \int_{0}^{\infty} \kappa^{2} E_{11}(\kappa) \mathrm{d} \kappa$

where the one-dimensional wave number $(\kappa)$ equals $2 \pi f / \bar{U}$ for a frequency $f$, and $E_{11}$ represents the spectral density of $u$. Antonia demonstrates that high frequency electronic noise in the hot-wire signal leads to a higher calculated dissipation rate than expected. The study herein quantifies the influence of the noise on the determined dissipation rate when using Eq. 4.

The fourth approach relies on determining $\varepsilon$ from a power decay law of turbulence kinetic energy. The turbulence kinetic energy $(q)$ equals $\overline{u^{2}}+\overline{v^{2}}+\overline{w^{2}}$. Studies of $q$ downstream of passive grids at Taylor Reynolds numbers less than 100 and turbulence intensities less than $3 \%$ have shown that:

$\frac{q / 3}{\bar{U}^{2}}=A_{q}\left(\frac{x}{M_{U}}-\frac{x_{o}}{M_{U}}\right)^{-n_{q}}$

where $A_{q}$ represents the decay coefficient, $M_{U}$ the grid rod spacing, $x_{o}$ the virtual origin, and $n_{q}$ the decay exponent. Studies by Comte-Bellot and Corrsin (1966), Warhaft and Lumley (1978), Mohamed and LaRue (1990), Antonia et al. (2003), Lavoie et al. (2005), Krogstad and Davidson (2010), Kitamura et al. (2014), and Kamruzzaman et al. (2014) found a virtual origin of $-2 \leq x_{o} / M_{U} \leq 7.3$ and a decay exponent of $1.10 \leq n_{q} \leq 1.36$ for passive grid generated turbulence. For active grid generated turbulence, Makita and Sassa (1991) determined $n_{q}=1.43$ with $x_{o} / M_{U}=-12$ , whereas Mydlarski and Warhaft (1996), Kang et al. (2003), and Mordant (2008) assume $x_{o} / M_{U}=0$ to determine $1.21 \leq n_{q} \leq 1.25$.
For homogeneous and isotropic flow upon the application of Taylor's Hypothesis (Tennekes and Lumley 1972; Pope 2000):

$\frac{\partial}{\partial t}\left(\frac{3}{2} q\right)=-\bar{U} \frac{\partial}{\partial x}\left(\frac{3}{2} q\right)=-\varepsilon$

Combining Eqs. 5 and 6 yields an expression for $\varepsilon$ as:

$\varepsilon=\frac{3}{2} \frac{n_{q} A_{q} \bar{U}^{3}}{M_{U}}\left(\frac{x}{M_{U}}-\frac{x_{o}}{M_{U}}\right)^{-n_{q}-1}$

Kang et al. (2003), in a flow downstream of an active grid for $20 \leq x / M_{U} \leq 48$ at Taylor Reynolds numbers $\left(R_{\lambda}\right)$ between 626 and 716, present a comparison of the dissipation rate obtained using a third-order structure function and the corresponding dissipation rate obtained from Eq. 7 with $x_{o} / M_{U}=0, A_{q}=1.80, n_{q}=1.25$, and an ensemble average mean velocity of $11.2 \mathrm{~m} \mathrm{~s}^{-1}$. The dissipation rate from the third-order structure function differed between 1 and $9 \%$ when compared to the dissipation from Eq. 7. The study reported herein utilizes the $A_{q}$ and $n_{q}$ determined by Koster (2018) for both a zero virtual origin and an optimized virtual origin. Based on the methods of Antonia et al. (2003) and Kamruzzaman et al. (2014), Koster (2018) determined the virtual origin by finding the value of $x_{o}$ that leads to the largest range of downstream positions where $\lambda^{2} / M_{U}\left(x-x_{o}\right)$ remains constant, where:

$\lambda=\left(\frac{5 v q}{\varepsilon}\right)^{0.5}$

defines the Taylor microscale $(\lambda)$. The analysis seeks to show that the selection of the virtual origin impacts the calculated dissipation rate obtained from Eq. 7.

In summary, the study presented herein seeks to compare the values of the dissipation rate in a flow downstream of an active grid using analog and numerical differentiation of the time-resolved velocity, the integration of the velocity derivative power spectrum (Eq. 4), and the dissipation rate obtained using a power decay law of turbulence kinetic energy (Eq. 7). Section 2 outlines the experimental method used to collect the hot-wire data. A discussion of the filtering method used to process the data and the numerical differentiation schemes follows in Sect. 3. The study concludes with Sect. 4 showing comparisons of the results obtained using the four approaches to obtain the dissipation rate. 
Table 1 Characteristics of the turbulent flow at different nominal velocities

\begin{tabular}{llccll}
\hline $\begin{array}{l}\text { Nominal } \\
\text { velocity } \\
\left(\mathrm{m} \mathrm{s}^{-1}\right)\end{array}$ & $l(\%)$ & $L(\mathrm{~m})$ & $\begin{array}{l}\lambda \times 10^{3} \\
(\mathrm{~m})\end{array}$ & $\begin{array}{l}\eta \times 10^{4} \\
(\mathrm{~m})\end{array}$ & $R_{\lambda}$ \\
\hline 4 & $5.8-11.1$ & $0.119-$ & $9.84-$ & $3.07-4.94$ & $193-275$ \\
& & 0.222 & 14.13 & & \\
6 & $6.4-10.6$ & $0.135-$ & $9.15-$ & $2.46-3.53$ & $267-363$ \\
& & 0.177 & 11.65 & & \\
8 & $6.9-10.4$ & $0.149-$ & $8.28-$ & $2.01-2.73$ & $353-453$ \\
& & 0.187 & 10.23 & & \\
\hline
\end{tabular}

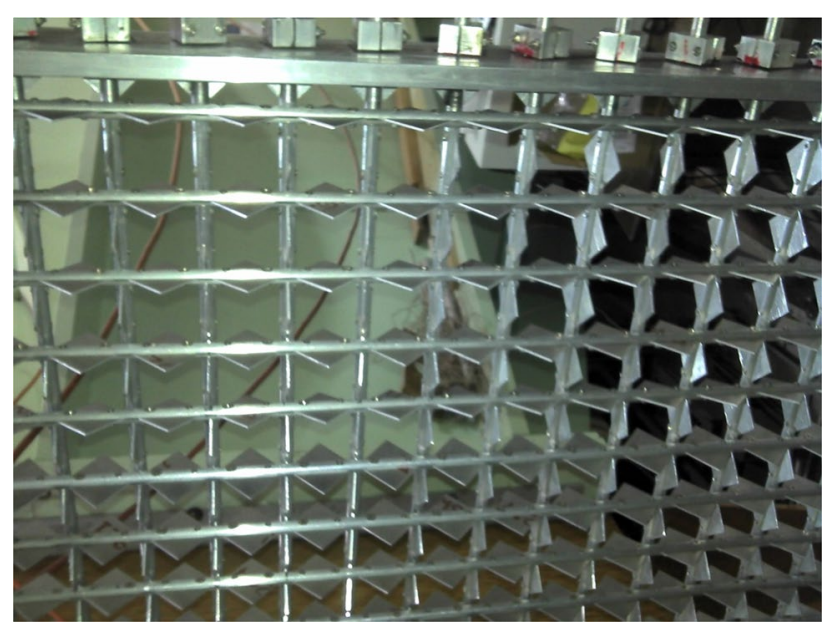

Fig. 1 Based on Makita's 1991 design (Makita and Sassa 1991) as implemented by Mydlarski and Warhaft (1996), the grid consists of 12 vertical and 18 horizontal rods with a diameter of $9.5 \mathrm{~mm}$ and a spacing $\left(M_{U}\right)$ of $50.4 \mathrm{~mm}$. One-hundred and eighty-seven diamond shaped flaps (34.3 mm edge length and $1.55 \mathrm{~mm}$ thickness), center mounted to the rods, complete the grid

\section{Experimental method}

The experimental setup consists of a closed return wind tunnel, an active grid, and several sensors. The closed return wind tunnel has a test section $0.61 \mathrm{~m}$ wide by $0.91 \mathrm{~m}$ high by $6 \mathrm{~m}$ long (cf. Selzer 2001; Puga and LaRue 2017). Downstream measurements begin $1.778 \mathrm{~m}$ from the active grid and extend to $5.46 \mathrm{~m}$ downstream of grid. The test section diverges to account for boundary layer growth, ensuring nearly constant nominal velocities of 4,6 , and $8 \mathrm{~m} \mathrm{~s}^{-1}$. The flow has the turbulence intensity $(l)$, integral length scale $(L)$, Taylor microscale, Kolmogorov length scale $(\eta)$, and Taylor Reynolds number $\left(R_{\lambda}=\bar{U} \lambda / v\right)$ shown in Table 1 .

The active grid generates moderate intensity turbulence by rotating finned rods at randomly determined rotation speed, direction, and rotation period (Fig. 1). Two independent Propeller Proto USB boards with P8X32-Q44
Propeller chips (Parallax Inc.: Rocklin, CA) control 15 Anaheim Automation 17MD102S-00 stepper motors (Anaheim, CA) each. For this experiment, the microcontrollers maintain a mean rotation rate of two revolutions per second with a $25 \%$ variance such that the rods can rotate at any speed between 1.5 and 2.5 revolutions per second. The period of rotation equals $250 \mathrm{~ms}$ with a $50 \%$ variance, such that the period ranges between 125 and $375 \mathrm{~ms}$. After completing the assigned period of rotation in one direction, the microcontrollers send a new, randomly determined rotation speed, direction, and rotation period within the stated variances. The random rotation rate minimizes the amplitude of a $4 \mathrm{~Hz}$ spike in the power spectra that corresponds to twice the rotation rate (Puga and LaRue 2017).

The collection of the data relies on three sensors. A pitot-static tube connected to an MKS Baratron Model 698A11TRE differential pressure transducer (MSK Instruments: Andover, MA) determines the mean velocity. A platinum resistance thermometer (PRT) made by Omega Engineering Inc. (Bridgeport, NJ) combined with a custom built Wheatstone bridge allows for correcting the hot-wire signal for temperature drift in the wind tunnel. The three sensors are mounted at the same vertical location on a traverse but displaced horizontally by $12 \mathrm{~mm}$ on either side of the hot-wire.

The data presented in this paper come from two different hot-wire sensors. For 4 and $6 \mathrm{~m} \mathrm{~s}^{-1}$, the hot-wire sensor has a length of $1 \mathrm{~mm}$ and a diameter of $5.08 \mu \mathrm{m}$, giving a nominal length to diameter ratio of 200 . To improve resolution, the $8 \mathrm{~m} \mathrm{~s}^{-1}$ experiment used a hot-wire $0.4 \mathrm{~mm}$ long with a $1.27 \mu \mathrm{m}$ diameter-with a nominal length to diameter ratio of 315 . Both sensors have an over-heat ratio of 1.75 resulting in the longer sensor having a frequency response of $18 \mathrm{kHZ}$ at a mean velocity of $18 \mathrm{~m} \mathrm{~s}^{-1}$ and the shorter sensor having a frequency response of $20 \mathrm{kHz}$ at $24 \mathrm{~m} \mathrm{~s}^{-1}$, based on the square-wave response. An AA Lab Systems (Westminster, CA) AN-1005 constant temperature anemometer (CTA) controls the long sensor and a custom build CTA controls the shorter sensor. Valente and Vassilicos (2011) demonstrated the AN-1005 produces an underestimation of the dissipation rate as a result of an issue with the built-in square-wave test in the unit used. While the AN-1005 used in this work could have the same problem, the analysis focuses on comparing ratios of the dissipation rates calculated from the same signal. Therefore, the different dissipation rates will have the same underestimation and cancel out in the ratios presented.

The voltage signal of the CTA undergoes an initial filtering to limit the influence of electronic noise to less than $3 \%$ of the root-mean-square of the time averaged temporal velocity derivative $\left(\overline{(\partial u / \partial t)^{2}}\right)$. The corner frequency $\left(f_{c}\right.$ )—defined as the $3 \mathrm{~dB}$ point—of the filter approximately equals $2-4$ times the Kolmogorov frequency. The signal 


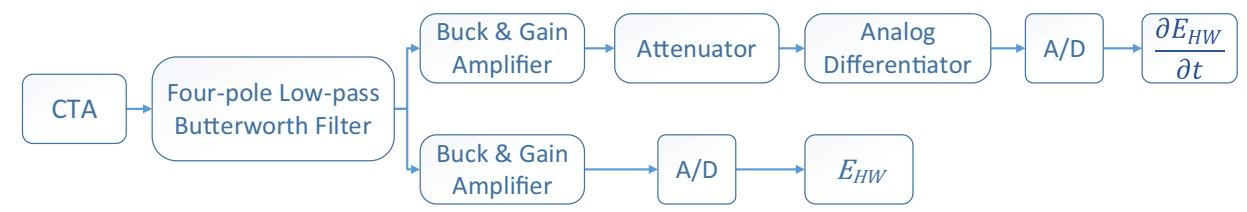

Fig. 2 Signal processing produces $\frac{\partial E_{H W}}{\partial t}$ and $E_{H W}$ from the same signal, allowing the determination of the temporal derivative of velocity from Eq. 10, which requires both variables

then splits into two different paths (Fig. 2) producing the time-resolved hot-wire voltage $\left(E_{H W}\right)$ and the time-resolved temporal-derivative of the hot-wire voltage $\left(\frac{\partial E_{H W}}{\partial t}\right)$. A measurement-computing 16-bit USB-1608HS (Norton, MA) analog-to-digital (A/D) converter sampling at 2.3 times the corner frequency over a \pm 10 volt input range sends the data to a personal computer. The amplifiers and analog differentiator have frequency responses of $70 \mathrm{kHz}$ and $18 \mathrm{kHz}$, respectively.

Converting $E_{H W}$ at each downstream position into the time resolved velocity, $U$, follows the temperature-corrected form of King's Law shown in Eq. 9.

$\frac{E_{H W}^{2}}{T_{w}-T_{g}}=A_{H W}+B_{H W} U^{n}$

In Eq. 9, $T_{w}$ and $T_{g}$ equal the average temperature of the wire and the temperature of the gas, respectively. A calibration of the sensors as a function of temperature and velocity determine $T_{w}$ and the correlation exponent ( $n$ ) based on the method of least squares. For $4 \mathrm{~m} \mathrm{~s}^{-1}, T_{w}=249.9^{\circ} \mathrm{C}$ and $n=0.403$. At $6 \mathrm{~m} \mathrm{~s}^{-1}, T_{w}=249.9^{\circ} \mathrm{C}$ and $n=0.403$. For $8 \mathrm{~m} \mathrm{~s}^{-1}, T_{w}=243.0^{\circ} \mathrm{C}$ with the standard value of $n=0.45$. A calibration before and after data collection determines the calibrations constants $A_{H W}$ and $B_{H W}$. The data presented herein correspond to only data collected where the statistics - mean, variance, skewness, and kurtosis - of the velocity and velocity derivative differ by less than $2 \%$ between the two calibrations.

Similarly, for the temporal velocity derivative $(\partial U / \partial t)$ :

$\frac{\partial U}{\partial t}=\frac{1}{n}\left[\frac{2 E_{H W}}{B_{H W}\left(T_{w}-T_{g}\right)} \frac{\partial E_{H W}}{\partial t}\right]\left(\frac{E_{H W}^{2}}{B_{H W}\left(T_{w}-T_{g}\right)}-\frac{A_{H W}}{B_{H W}}\right)^{\frac{1}{n}-1}$

where Eq. 10 results from the temporal derivative of Eq. 9.

To ensure the instantaneous flow angles did not exceed $33^{\circ}$ for cross-wire measurements, Koster (2018) limited the starting point of the analysis to $x / M_{U}$ values of 60,75 , and 80 for 4,6 , and $8 \mathrm{~m} \mathrm{~s}^{-1}$, respectively. The study presented herein utilizes the same starting points. The data consists of $120 \mathrm{~s}$ samples at each downstream position.

\section{Filtering and numerical differentiation}

The presence of electronic noise in the signal can influence the measured value of $\varepsilon$ and thus impact the comparison between the different approaches to calculate the dissipation rate. Furthermore, the accurate assessment of the accuracy of the numerical differentiation approach to determine the dissipation rate depends on the order of the finite centraldifference scheme as noted by Hearst et al. (2012). This section discusses the different central-difference schemes used to calculate the temporal derivative of the velocity, the noise present in the signals, and the digital filtering method implemented to address the electronic noise - which also employs numerical differentiation.

\subsection{Finite central-difference schemes}

The accuracy of the computed dissipation rate depends on the order of the central difference scheme (Hearst et al. 2012). This study herein presents the relative accuracy of a 5-point, 9-point, and 23-point finite central-difference scheme. The equations to determine the derivative of a function $f$, defined as $f^{\prime}$, for the $n$th sample of a discretized time-series follows Hearst et al. (2012) for the 5-point and 9-point finite central-difference schemes. The 23-point finite central-difference scheme has the form:

$$
\begin{aligned}
f_{n}^{\prime}\left(t_{n}\right) \Delta t= & -\mathrm{a} f\left(t_{n}-11 \Delta t\right)+\mathrm{b} f\left(t_{n}-10 \Delta t\right)-\mathrm{c} f\left(t_{n}-9 \Delta t\right) \\
& +\mathrm{d} f\left(t_{n}-8 \Delta t\right)-\mathrm{e} f\left(t_{n}-7 \Delta t\right)+\mathrm{f} f\left(t_{n}-6 \Delta t\right) \\
& -\mathrm{g} f\left(t_{n}-5 \Delta t\right)+\mathrm{h} f\left(t_{n}-4 \Delta t\right)-\mathrm{i} f\left(t_{n}-3 \Delta t\right) \\
& +\mathrm{j} f\left(t_{n}-2 \Delta t\right)-\mathrm{k} f\left(t_{n}-\Delta t\right)+\mathrm{k} f\left(t_{n}+\Delta t\right) \\
& -\mathrm{j} f\left(t_{n}+2 \Delta t\right)+\mathrm{i} f\left(t_{n}+3 \Delta t\right)-\mathrm{h} f\left(t_{n}+4 \Delta t\right) \\
& +\mathrm{g} f\left(t_{n}+5 \Delta t\right)-\mathrm{f} f\left(t_{n}+6 \Delta t\right)+\mathrm{e} f\left(t_{n}+7 \Delta t\right) \\
& -\mathrm{d} f\left(t_{n}+8 \Delta t\right)+\mathrm{c} f\left(t_{n}+9 \Delta t\right)-\mathrm{b} f\left(t_{n}+10 \Delta t\right) \\
& +\mathrm{a} f\left(t_{n}+11 \Delta t\right)
\end{aligned}
$$

where $t_{n}$ indicates the central point and $\Delta t$ the spacing of the points. The sampling rate determines $\Delta t$. Table 2 contains the coefficients for Eq. 11 determined by solving the system of 23 equations formed from the Taylor-series expansion about $t_{n}$ to the 22nd-order derivative for each of the 23 
Table 2 Coefficients for the 23-point finite central-difference equation (Eq. 11) shown to 4 decimal places

\begin{tabular}{ll}
\hline Coefficient & Value \\
\hline $\mathrm{a}$ & $1.2887 \times 10^{-7}$ \\
$\mathrm{~b}$ & $3.1187 \times 10^{-6}$ \\
$\mathrm{c}$ & $3.6384 \times 10^{-5}$ \\
$\mathrm{~d}$ & $2.7288 \times 10^{-4}$ \\
$\mathrm{e}$ & $1.4814 \times 10^{-3}$ \\
$\mathrm{f}$ & $6.2217 \times 10^{-3}$ \\
$\mathrm{~g}$ & $2.1154 \times 10^{-2}$ \\
$\mathrm{~h}$ & $6.0440 \times 10^{-2}$ \\
$\mathrm{i}$ & $1.5110 \times 10^{-1}$ \\
$\mathrm{j}$ & $3.5256 \times 10^{-1}$ \\
$\mathrm{k}$ & $9.1667 \times 10^{-1}$ \\
\hline
\end{tabular}

points (cf. Moin 2010). The schemes have truncation errors of $O\left(\Delta t^{4}\right), O\left(\Delta t^{8}\right)$, and $O\left(\Delta t^{22}\right)$ for the 5-point, 9-point, and 23-point scheme, respectively.

\subsection{Digital filtering method}

The hot-wire signal passes through an analog low-pass Butterworth filter having a $3 \mathrm{~dB}$ frequency set to $2-4$ times the Kolmogorov frequency as noted in Sect. 2. This filtering ensures that the filter has a negligible effect on the digitized velocity signal at frequencies at and below the Kolmogorov frequency. However, the signal does contain an amount of electronic noise. For example, a representative unfiltered power spectra of the temporal velocity derivative (Fig. 3) shows an increasing power spectra at high frequencies instead of a continual decrease-indicating the presence of electronic noise. Figure 4 shows a representative unfiltered power spectra of the velocity, in which the noise becomes less apparent.

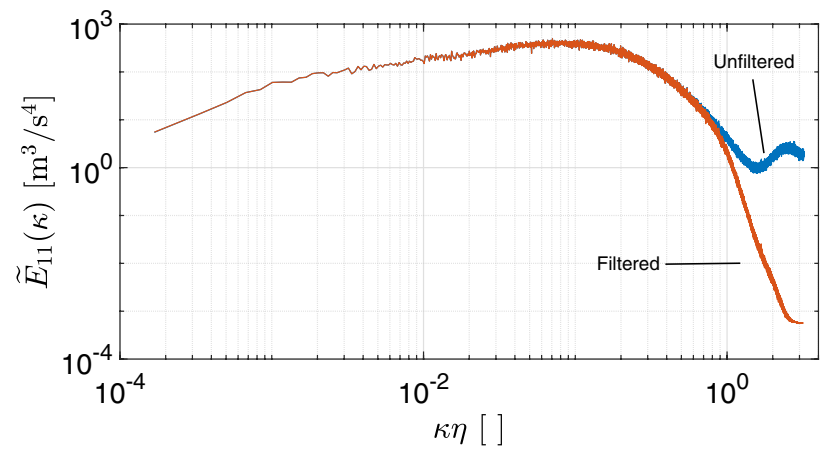

Fig. 3 Temporal velocity derivative power spectra $\left(\widetilde{E}_{11}\right)$ for an $R_{\lambda}=438$ at $x / M_{U}=91\left(8 \mathrm{~m} \mathrm{~s}^{-1}\right)$ demonstrate the difference between the unfilter signal (blue) which shows a secondary roll-off resulting from noise in the signal and the digitally filtered signal (orange)

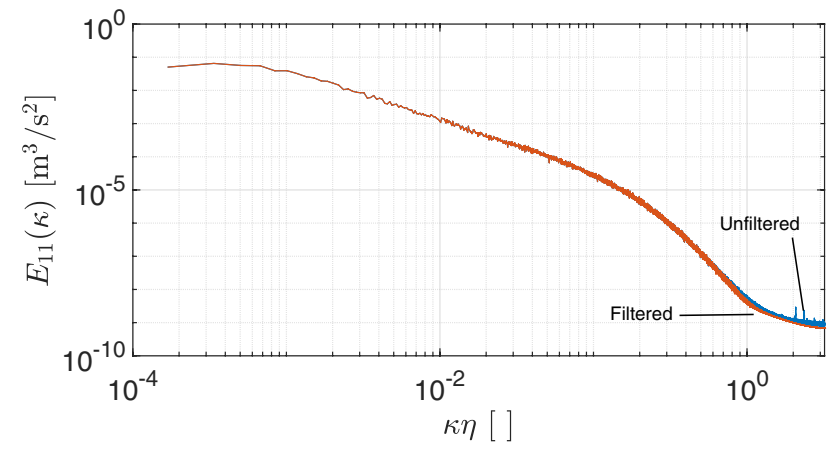

Fig. 4 Unlike the power spectra for the temporal derivative of velocity, the velocity power spectra $\left(E_{11}\right)$ for $R_{\lambda}=438$ at $x / M_{U}=91$ $\left(8 \mathrm{~m} \mathrm{~s}^{-1}\right)$ does not indicate a significant difference between the filtered (orange) and the unfiltered (blue) spectra

The application of a digital filter reduces the high-frequency noise present in the signals. The filtering scheme, applied in LabView, follows the iterative approach of Mi et al. (2011) with a few modifications. Figure 5 summarizes the process. An initial guess of half the sampling rate $(S)$ minus 1 for the corner frequency ( $f_{c}$, also noted as the $3 \mathrm{~dB}$ point) of a digital Butterworth filter starts the iterative process. Note that the velocity signal $\left(U^{(0)}\right)$ and the temporal derivative of the velocity signal determined from analog differentiation $\left((\partial u / \partial t)_{a}\right)$ each have a digital Butterworth filter but set to the same corner frequency as determined by the iterative scheme. The filtered velocity signal passes through a numerical differentiator to calculate the temporal derivative of the velocity $\left((\partial u / \partial t)_{n}\right)$ to determine its variance $\left(\overline{(\partial u / \partial t)_{n}^{2}}\right)$. The mean of the filtered velocity signal provides $\bar{U}$. Equations 1 and 2 then determine the dissipation rate of turbulence kinetic energy using the numerically differentiated velocity. Combining the calculated $\varepsilon$ and the kinematic viscosity $(v)$ of air in Eq. 12 determines the Kolmogorov length scale $(\eta)$.

$\eta=\left(\frac{v^{3}}{\varepsilon}\right)^{\frac{1}{4}}$

The algorithm then determines the Kolmogorov (1941) frequency $\left(f_{\eta}\right)$ defined as:

$f_{\eta} \equiv \frac{\bar{U}}{2 \pi \eta}$

As noted by Mi et al. (2011), the parameters $\varepsilon, \eta$, and $f_{\eta}$ change after applying the filter. The next iteration sets $f_{c}$ equal to the $f_{\eta}$ determined from the previous iteration. Each iteration re-filters the unfiltered velocity signal and temporal derivative of the velocity signal determined from analog differentiation instead of re-filtering the filtered signal as done by Mi et al. (2011). The iteration process concludes when 
Fig. 5 An iterative filtering scheme filters the original $U^{(0)}(t)$ and $\left(\frac{\partial u}{\partial t}\right)_{a}$ signal for each iteration based on the Kolmogorov frequency calculated from the previous iteration until convergence. The final iteration, $N$, also provides the filtered temporal derivative of the velocity from numerical differentiation

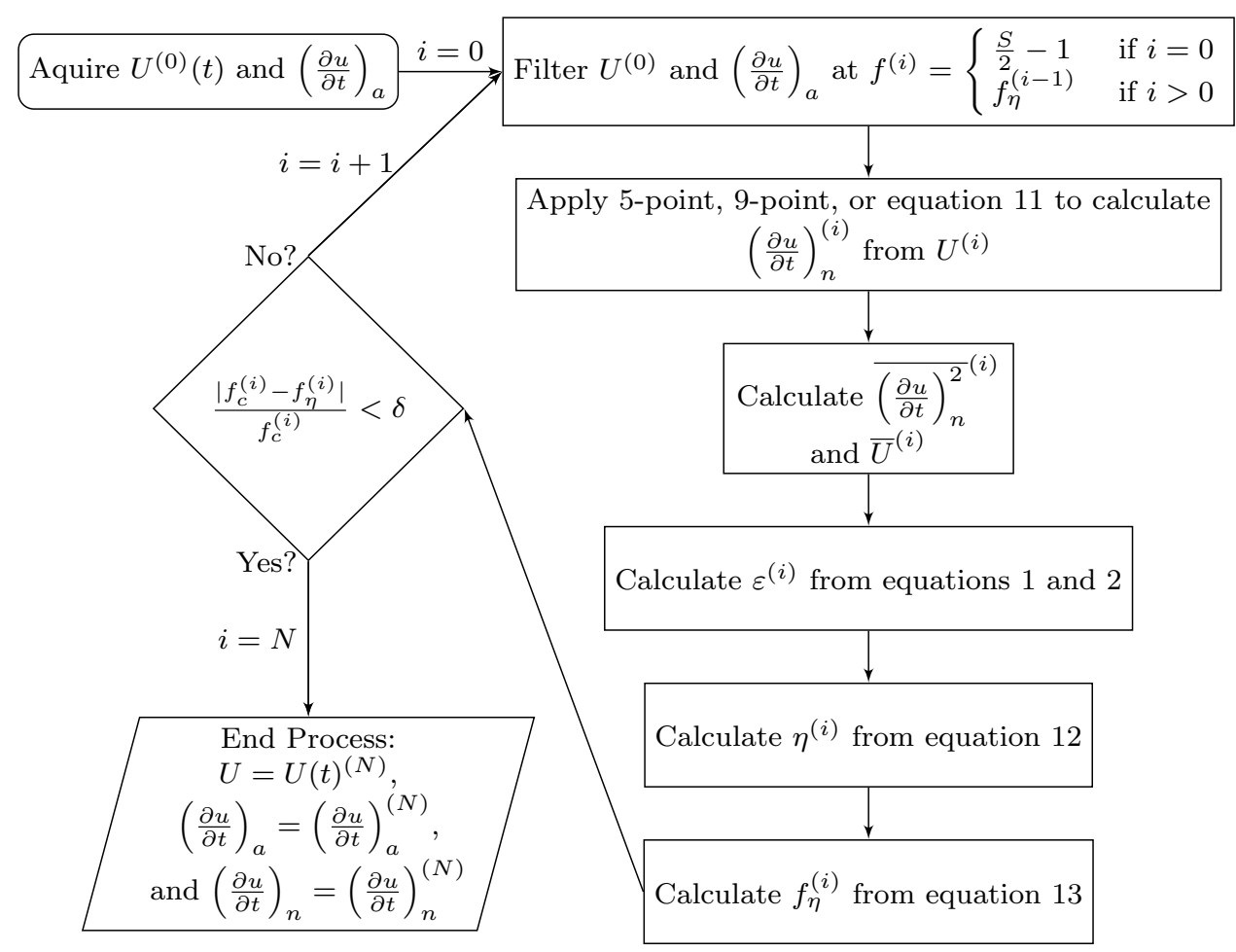

$\frac{\left|f_{c}^{(i)}-f_{\eta}^{(i)}\right|}{f_{c}^{(i)}}<\delta$

where $i$ indicates the iteration number and the convergence tolerance $(\delta)$ equals $1.0 \times 10^{-3}$. Stricter convergence tolerances showed statistically insignificant differences in the result $(\ll 0.1 \%)$. Figures 3 and 4 show the resulting power spectra after applying the filtering method. The results section discusses the effect of the order of the Butterworth filter and the order of the numerical differentiation on the computed dissipation rate.

\section{Results and discussion}

\subsection{Filter type, corner frequency, and integration order}

The first step in determining the dissipation rate of turbulence kinetic energy requires digital filtering. The selection of the corner frequency and the type of low-pass Butterworth filter could change the results. Since the determination of the corner frequency relies on the numerical differentiation of the hot-wire signal, this also plays a role in the filtering characteristics. Processing the data using a digital 3-pole, 5-pole, and 9-pole Butterworth filter for the three differentiation schemes (5-point, 9-point, and 23-point) showed that changing the number of poles has minimal impact. The percent difference relative to the 3-pole Butterworth increased by less than $0.5 \%$ for the numerically determined $\varepsilon$ and the $\varepsilon$ from the analog differentiator-henceforth referred to as $\varepsilon_{n}$ and $\varepsilon_{a}$, respectively. The percent difference of the ratio of $\varepsilon_{n}$ to $\varepsilon_{a}$ fell in a range of $\pm 0.05 \%$ relative to the 3-pole Butterworth filter. This held true for 4,6 , and $8 \mathrm{~m} \mathrm{~s}^{-1}$. Therefore, increasing the order of the Butterworth filter did not significantly change the results. The presented results use the digital 3-pole low-pass Butterworth filter.

The filtering scheme uses the iteratively determined Kolmogorov frequency as the corner frequency of the Butterworth filter. The choice of the Kolmogorov frequency as the corner frequency does not have a fundamental justification. Therefore, the analysis requires assessing the effect of the choice of the corner frequency. Setting the corner frequency to $90 \%, 110 \%$, and $150 \%$ of Kolmogorov frequency shows at most a $1.62 \%$ change in the calculated dissipation rates (for both the numerical and analog differentiation). Additionally, the ratio of $\varepsilon_{n}$ to $\varepsilon_{a}$ changes by at most $\pm 1 \%$. The stated percentages represent the change relative to the corner frequency equal to the Kolmogorov frequency and the 9-point central-difference scheme. The 5-point and 23-point central-difference schemes produced similar results. Since the determined dissipation rates and the corresponding ratio change by less than $2 \%$, the filtering of the presented results uses a corner frequency equal to the Kolmogorov frequency. 
After selecting a 3-pole low-pass Butterworth filter with a corner frequency equal to the Kolmogorov frequency to perform the analysis, the analysis considers the impact of the finite central-difference scheme order when calculating the temporal velocity derivative. Using the 9-point central difference scheme as the reference, a 5-point scheme produces a ratio of the dissipation rates $\left(\varepsilon_{n} / \varepsilon_{a}\right)$ at most $0.3 \%$ smaller than the value using the 9-point scheme. Conversely, the 23-point scheme leads to values of $\varepsilon_{n} / \varepsilon_{a}$ at most $0.02 \%$ larger than those found using the 9-point scheme. The small percent differences for both the 5-point and 23-point schemes relative to the 9-point scheme indicates that any of the three schemes would minimally impact the results. However, the 23-point scheme produces percent differences at least one order of magnitude smaller than the percent differences of the 5-point scheme. To further demonstrate the diminishing improvement of the higher order schemes, $\varepsilon_{n} / \varepsilon_{a}$ calculated using a 9-pole Butterworth filter with a 23-point central difference scheme differs by $\pm 0.02 \%$ relative to the 3 -pole Butterworth filter with a 9-point central-difference scheme-both with a corner frequency equal to the Kolmogorov frequency. Consequently, the presented results utilize a 9-point finite central-difference scheme.

In summary, the following results use digital filtering with a 3-pole low-pass Butterworth filter with a corner frequency equal to the Kolmogorov frequency and the numerical differentiation of the hot-wire signal obtained using a 9-point central-difference scheme.

\subsection{Dissipation rate from the analog differentiator}

The observation that hot-wires with a length greater than the Kolmogorov length scale have attenuated spectral values at high wavernumbers and a corresponding reduced dissipation rate (Wyngaard 1968) motivates a correction to the spectra. Applying the Wyngaard correction iteratively until the computed dissipation rate changes by less than $1 \%$ relative to the previous iteration, corrects the data for wire-length averaging. The application of Champagne's correction (Eq. 3) using the ensemble averages-denoted by $<>$ - shown in Table 3 with $\overline{u^{2}}$ and $\bar{U}$ at each downstream location corrects for the moderate intensity turbulence. Figure 6 shows the resulting normalized dissipation rate determined from the

Table 3 Ensemble average of the isotropy ratios $I_{u v}$ and $I_{u w}$ from Koster (2018)

\begin{tabular}{lll}
\hline Mean velocity $\left(\mathrm{m} \mathrm{s}^{-1}\right)$ & $\left\langle I_{u v}\right\rangle=\left\langle\overline{v^{2}} / \overline{u^{2}}\right\rangle$ & $\left\langle I_{u w}\right\rangle=\left\langle\overline{w^{2}} / \overline{u^{2}}\right\rangle$ \\
\hline 4 & 1.19 & 1.06 \\
6 & 1.09 & 1.18 \\
8 & 1.25 & 1.24 \\
\hline
\end{tabular}

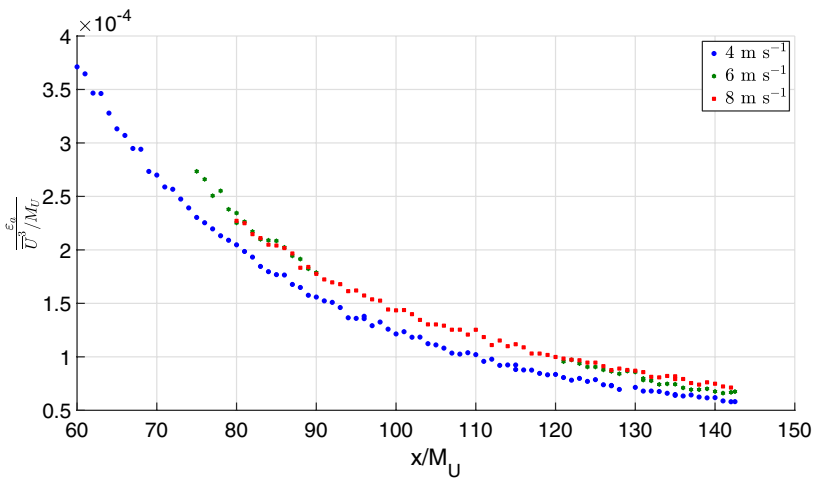

Fig. 6 Dissipation rate normalized by $\frac{\bar{U}^{3}}{M_{U}}$ for 4,6 , and $8 \mathrm{~m} \mathrm{~s}^{-1}$ determined from the analog differentiator and Eq. 10 corrected for wire length averaging and moderate intensity turbulence. The analysis does not include data for $6 \mathrm{~m} \mathrm{~s}^{-1}$ in the range of $90 \leq x / M_{U} \leq 120$ as the spectra of the velocity and the temporal velocity derivative show a noise peak uncharacteristic of electronic noise and not present in any of the other data

analog differentiator with a value of $1.568 \times 10^{-5} \mathrm{~m}^{2} \mathrm{~s}^{-1}$ for the kinematic viscosity of air.

\subsection{Comparison of numerical differentiation to analog differentiation}

The numerical differentiation determines a dissipation rate within $\pm 4 \%$ of the value from the analog differentiation (Fig. 7). Furthermore, the numerical differentiation overestimates the dissipation rate at 4 and $6 \mathrm{~m} \mathrm{~s}^{-1}$, while underestimating the dissipation rate at $8 \mathrm{~m} \mathrm{~s}^{-1}$.

The numerical differentiation over-estimating the dissipation rate does not make physical sense as numerical differentiation acts as a low-pass filter (Fig. 8). Therefore, the numerical differentiation should attenuate higher frequencies while having no effect on lower frequencies. Figure 9 shows

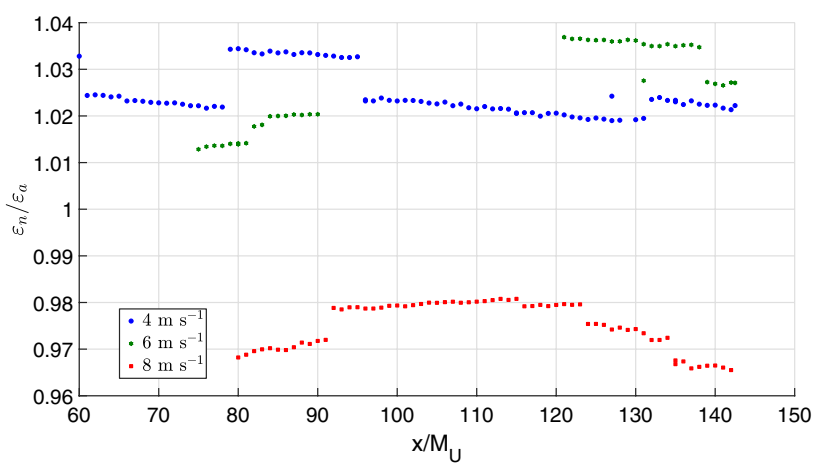

Fig. 7 Ratio of $\varepsilon_{n}$ to $\varepsilon_{a}$ versus $x / M_{U}$ for 4,6 , and $8 \mathrm{~m} \mathrm{~s}^{-1}$. The jumps in the data for a given velocity coincide with changes in the amplification of the signal 


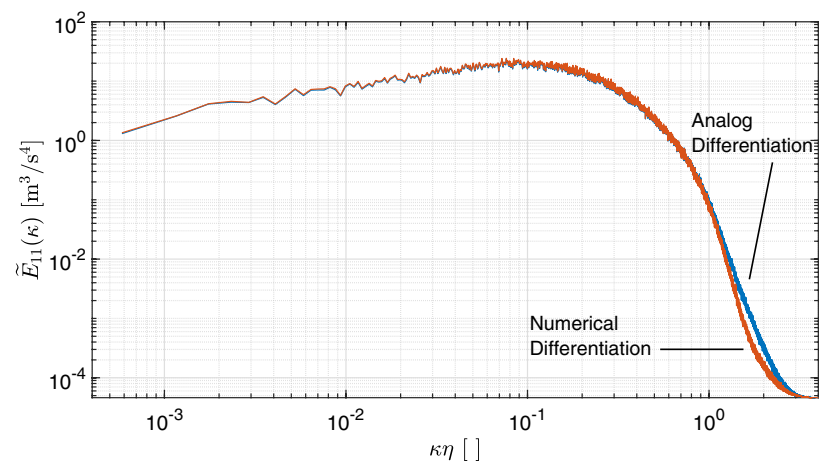

Fig. 8 Numerical differentiation acts as a low-pass filter resulting in a higher attenuation of the numerically determined temporal velocity derivative power spectrum (orange) at large $\kappa \eta$ as compared to analog differentiation (blue) as demonstrated for $4 \mathrm{~m} \mathrm{~s}^{-1}$ at $x / M_{U}=81$ $\left(R_{\lambda}=249\right)$

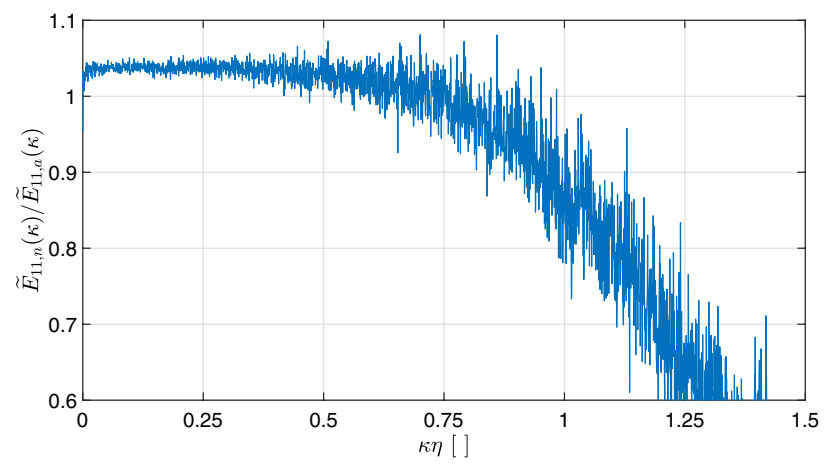

Fig. 9 Ratio of the temporal derivative of velocity spectra from numerical differentiation to that of analog differentiation does not equal one at $\kappa \eta<0.5$, indicating a mismatch between the amplifier gains on the velocity and analog differentiator channels as shown for $4 \mathrm{~m} \mathrm{~s}^{-1}$ at $x / M_{U}=81\left(R_{\lambda}=249\right)$

the ratio of the temporal velocity derivative power spectra for numerical $\left(\widetilde{E}_{11, n}\right)$ and analog differentiation $\left(\widetilde{E}_{11, a}\right)$ shown in Fig. 8. The ratio in Fig. 9 clearly indicates that spectral values in the range $0<\kappa \eta<0.5$ for the numerical differentiation have a larger value than the corresponding spectral values for the analog differentiation. Additionally, the magnitude of the ratio remains relatively constant in this range. Therefore, the mismatch must result from uncertainty in the amplifier gains.

Averaging the values over the largest extent of the flat region in Fig. 9 determines a correction factor of the power spectra of the numerical temporal derivative of the velocity. Applying the correction factor to $\widetilde{E}_{11, n}$ demonstrates how the experimental error impacts the ratio of dissipation rates (Fig. 10). The experiments at 4 and $6 \mathrm{~m} \mathrm{~s}^{-1}$ use the same

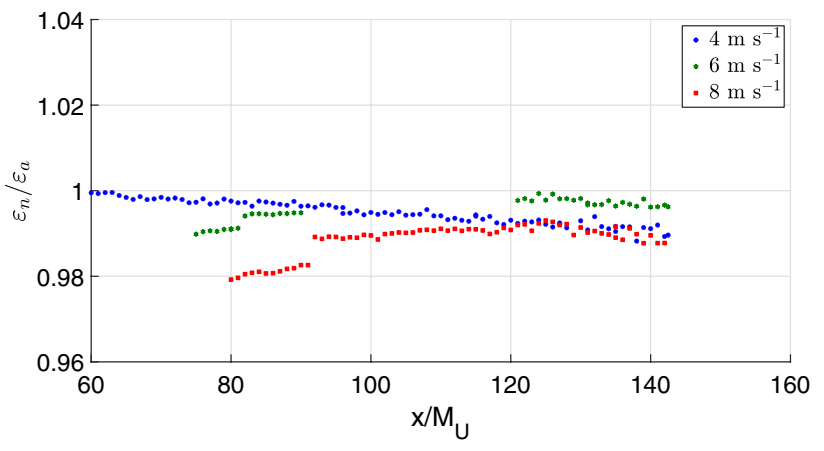

Fig. 10 After correcting for a gain mismatch, the ratio of $\varepsilon_{n}$ to $\varepsilon_{a}$ versus $x / M_{U}$ becomes less than 1 for 4,6 , and $8 \mathrm{~m} \mathrm{~s}^{-1}$

channel of the amplifier, whereas $8 \mathrm{~m} \mathrm{~s}^{-1}$ uses a different amplifier. The analysis shows that for 4 and $6 \mathrm{~m} \mathrm{~s}^{-1}$, the spectra should decrease by $3 \%$ on average. Conversely for $8 \mathrm{~m} \mathrm{~s}^{-1}$, the spectra should increase by $1 \%$ on average. After correcting for the assumed amplifier gain error, the $\varepsilon_{n}$ becomes $0-2.5 \%$ lower than $\varepsilon_{a}$ (Fig. 10). The scale of Fig. 10 makes it appear that the $4 \mathrm{~m} \mathrm{~s}^{-1}$ set has a decaying trend with increasing downstream distance but with only a $1 \%$ variation.

\subsection{Determination of the dissipation rate obtained by integrating the temporal velocity derivative spectra obtained from the velocity spectra}

The preceding section presented a comparison of the dissipation rates obtained using numerical differentiation and analog differentiation. This section presents a comparison between the third method of determining the dissipation rate-defined by Eq. 4-and the analog differentiation method. Antonia et al. (2003) notes that high frequency noise plays a role in the magnitude of the determined dissipation rate when using Eq. 4. Consequently, the analysis of this method utilizes the unfiltered velocity and analog temporal velocity derivative signals. As the application of the Wyngaard correction would only amplify the high frequency noise of the unfiltered signal, the analysis uses the uncorrected data. Figure 11 shows a comparison between the power spectra of the temporal velocity derivative determined from the analog differentiator, the numerical differentiator, and by multiplying the velocity spectral density by $\kappa^{2}\left(\kappa^{2} E_{11}\right.$ in Eq. 4). Additionally, Fig. 11 shows the velocity power spectra, $E_{11}$.

In Fig. 11, the $\kappa^{2} E_{11}$ method determines the power spectra of the temporal velocity derivative similar to the analog and numerical differentiators up to $\kappa \eta=0.8$. After $\kappa \eta=0.8$, the $\kappa^{2} E_{11}$ method amplifies the noise present in the velocity power spectra ( $E_{11}$ in Fig. 11$)$. Consequently, the $\kappa^{2} E_{11}$ method produces spectra values larger than the analog 


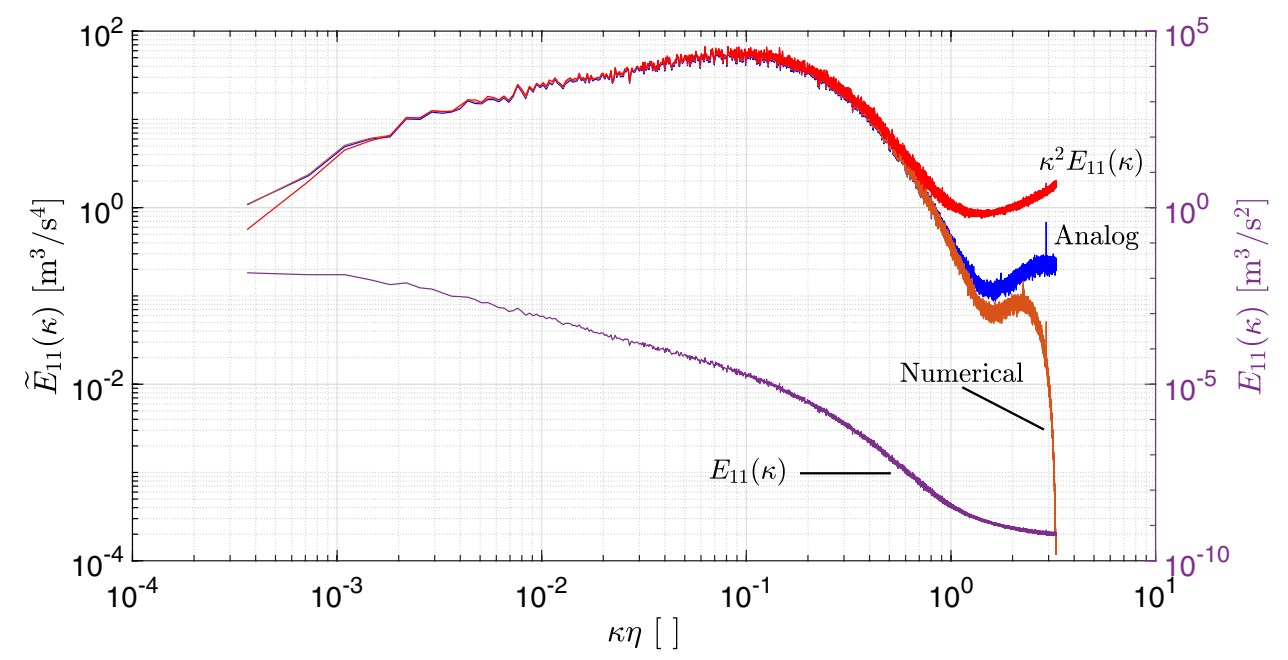

Fig. 11 For $6 \mathrm{~m} \mathrm{~s}^{-1}$ at $x / M_{L}=126\left(R_{\lambda}=279\right)$, the temporal-derivative of velocity spectrum $\left(E_{11}\right)$ calculated by the $\kappa^{2} E_{11}(\kappa)$ method (red) shows an influence of noise nearly one decade larger than the noise present in the analog (blue) or numerical (orange) differentiation methods. This occurs despite the velocity spectra $\left(E_{11}\right.$, purple)

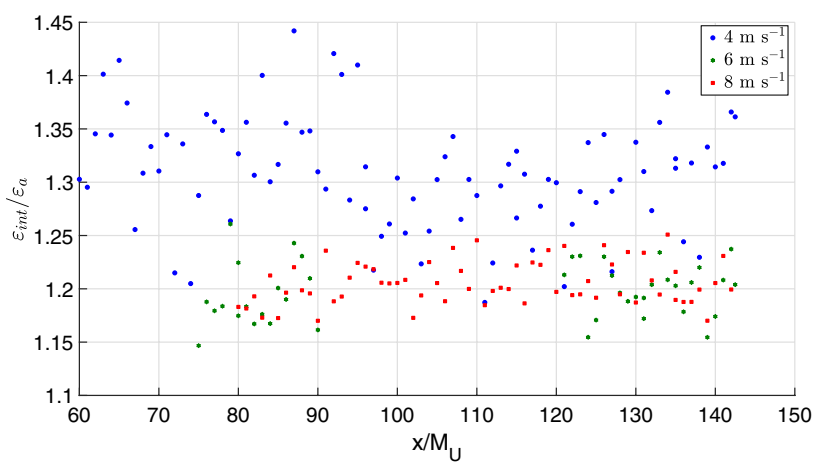

Fig. 12 Ratio of $\varepsilon_{\text {int }}$ to $\varepsilon_{a}$ versus $x / M_{U}$ for 4,6 , and $8 \mathrm{~m} \mathrm{~s}^{-1}$

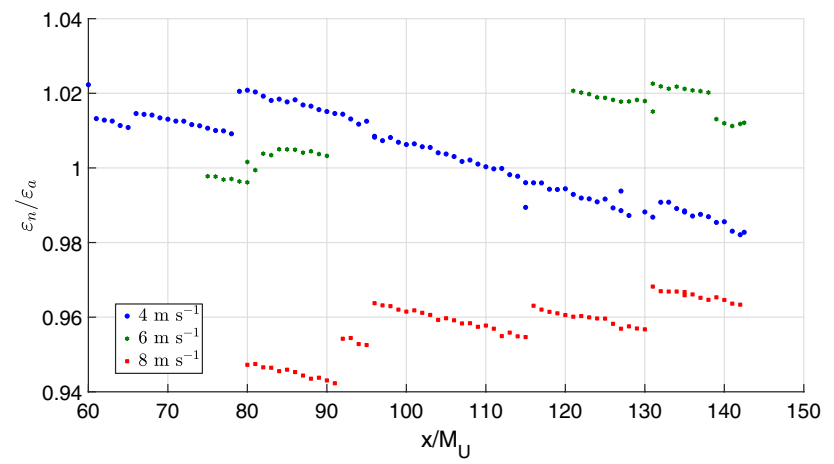

Fig. 13 Ratio of $\varepsilon_{n}$ to $\varepsilon_{a}$ versus $x / M_{U}$ for 4,6 , and $8 \mathrm{~m} \mathrm{~s}^{-1}$ based on the unfiltered signals. The figure excludes the $6 \mathrm{~m} \mathrm{~s}^{-1}$ data at an $X / M_{U}$ of 119 and 120 which fall at $\varepsilon_{n} / \varepsilon_{a}=1.17$. The jumps in the data for a given nominal velocity correspond to changes in the amplification of the signal and no clear explanation arises for the trend of the data not appearing to have a significant amount of noise. The power spectra of the numerical temporal derivative of the velocity indicates the filtering affect of numerical differentiation causing the spectral values to rapidly decrease starting at $\kappa \eta=2.5$

differentiation method beyond $\kappa \eta=0.8$. The amplification of the noise when applying Eq. 4 results in a dissipation rate, $\varepsilon_{i n t}, 14-45 \%$ larger relative to $\varepsilon_{a}$ (Fig. 12). The overall characteristics of the spectra in Fig. 11 remain the same for different downstream locations and mean velocities.

To further demonstrate the sensitivity of the integration method to noise, Fig. 13 shows the ratio of the dissipation rate from numerical differentiation to the dissipation rate from analog differentiation for the unfiltered signals. Whereas the integration method leads to an over-estimation of the dissipation rate by $14-45 \%$, the numerical differentiation of the unfiltered signal determines $\varepsilon$ within -6 to $3 \%$ of the dissipation rate from analog differentiation. Comparing Figs. 7 and 13, the $\varepsilon_{n} / \varepsilon_{a}$ for the unfiltered data decreases by at most 0.001-0.04 compared to the filtered data. This small change between filtered and unfiltered $\varepsilon_{n} / \varepsilon_{a}$ indicates that the integration method over-estimating the dissipation rate by $14-45 \%$ relative to the analog differentiation results from the method's high sensitivity to high-frequency noise.

To achieve a result from Eq. 4 comparable to numerical differentiation requires careful treatment of the noise. Mydlarski and Warhaft (1996) utilized the integration method to determine the dissipation rate and compared the value to the dissipation rate calculated from a 5-point central-difference scheme (Mydlarski, private communication: 2019). To minimize the electronic noise in the signal, Mydlarski and Warhaft low-pass and high-pass the signal, allowing for the amplification of the high-frequency components beyond the noise (Mydlarski, private communication: 2019). With minimal noise in the signal, the integration of the velocity spectra by Eq. 4 calculates a dissipation rate 
Table 4 Coefficients for Eq. 7 with an assumed zero virtual origin

\begin{tabular}{llll}
\hline Velocity $\left(\mathrm{m} \mathrm{s}^{-1}\right)$ & 4 & 6 & 8 \\
\hline$A_{q}$ & 73.475 & 240.281 & 368.465 \\
$n_{q}$ & 1.492 & 1.525 & 1.456 \\
$\sigma_{q} \times 10^{2}$ & 2.35 & 2.56 & 2.31 \\
\hline
\end{tabular}

Table 5 Coefficients for Eq. 7 with a nonzero virtual origin

\begin{tabular}{llll}
\hline Velocity $\left(\mathrm{m} \mathrm{s}^{-1}\right)$ & 4 & 6 & 8 \\
\hline$A_{q}$ & 4759.085 & $14,398.419$ & $103,709.663$ \\
$n_{q}$ & 2.238 & 2.228 & 2.409 \\
$x_{o} / M_{U}$ & -39.41 & -46.16 & -67.88 \\
$\sigma_{q} \times 10^{2}$ & 2.27 & 2.88 & 2.12 \\
\hline
\end{tabular}

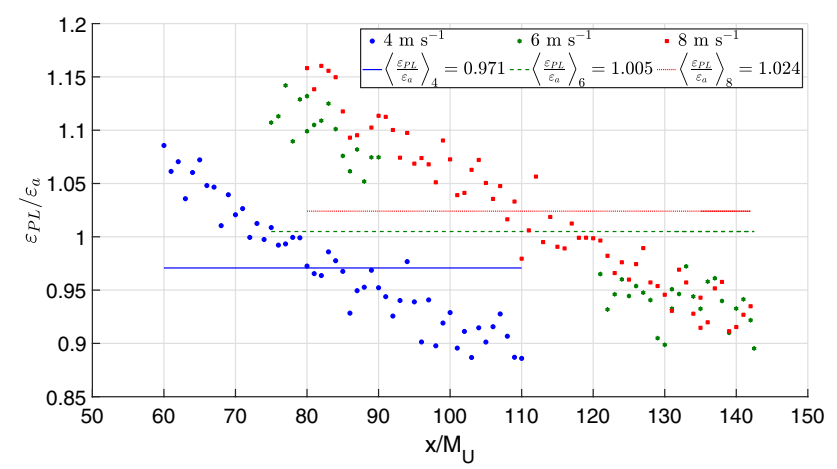

Fig. 14 Ratio between the dissipation rate calculated from the power law with a zero virtual origin $\left(\varepsilon_{P L}\right)$ and the analog differentiation $\left(\varepsilon_{a}\right)$ versus $x / M_{U}$ for 4,6 , and $8 \mathrm{~m} \mathrm{~s}^{-1}$

within 5\% of the dissipation rate calculated from the numerical differentiation (Mydlarski and Warhaft 1996).

\subsection{Influence of the virtual origin on determining the dissipation rate}

Koster (2018) determined the decay coefficient $\left(A_{q}\right)$ and the decay exponent $\left(n_{q}\right)$ using a nonlinear least-squares fit of the turbulence kinetic energy $(q)$ with an assumed zero virtual origin (Table 4). Additionally, Koster determined the virtual origin $\left(x_{o}\right), A_{q}$, and $n_{q}$ (Table 5) using the method outlined in section 1 . The coefficients in Tables 4 and 5 correspond to a fit of $q$ defined by Eq. 5 without the $\bar{U}^{2}$ scaling-consequently Eq. 7 becomes proportional to $\bar{U}$ instead of $\bar{U}^{3}$. The resulting power-law fits for $q$ show a similar standard deviation ( $\sigma_{q}$ in Tables 4 and 5), indicating both a nonzero virtual origin and a zero virtual origin result in equal fits to the data. The analysis of this section
Table 6 Comparison of the slope and standard deviation for linear fits of $\varepsilon_{P L} / \varepsilon_{a}$ with a zero and nonzero virtual origin

\begin{tabular}{|c|c|c|c|c|}
\hline \multirow{2}{*}{$\begin{array}{l}\text { Velocity } \\
\left(\mathrm{m} \mathrm{s}^{-1}\right)\end{array}$} & \multicolumn{2}{|l|}{$\frac{x_{o}}{\underline{M_{U}}}=0$} & \multicolumn{2}{|l|}{$\frac{x_{o}}{M_{U}} \neq 0$} \\
\hline & $\mathrm{m} \times 10^{3}$ & $\sigma \times 10^{2}$ & $\mathrm{~m} \times 10^{3}$ & $\sigma \times 10^{2}$ \\
\hline 4 & -3.6 & 1.64 & -0.062 & 1.73 \\
\hline 6 & -3.1 & 2.13 & -0.35 & 2.03 \\
\hline 8 & -3.71 & 1.76 & -0.34 & 2.04 \\
\hline
\end{tabular}

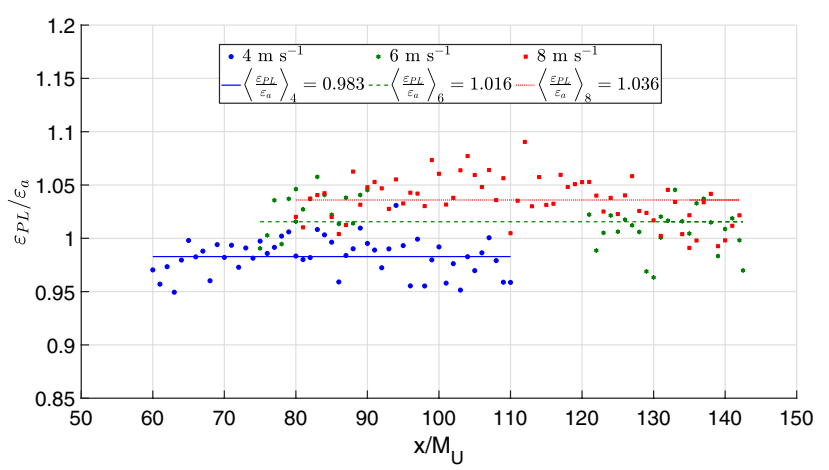

Fig. 15 Ratio between the dissipation rate calculated from the power law with a calculated virtual origin $\left(\varepsilon_{P L}\right)$ and the analog differentiation $\left(\varepsilon_{a}\right)$ versus $x / M_{U}$ for 4,6 , and $8 \mathrm{~m} \mathrm{~s}^{-1}$.

uses the coefficients in Tables 4 and 5 in Eq. 7 and the data analyzed by Koster (2018) to determine $\varepsilon_{P L}$ for comparison to $\varepsilon_{a}$.

With a $x_{o} / M_{U}$ equal to zero in Eq. 7 with the coefficients shown in Table 4, the ratio of the dissipation rate from the power law $\left(\varepsilon_{P L}\right)$ to $\varepsilon_{a}$ decreases with increasing downstream distance (Fig. 14). Conversely, the ensemble average of the ratios nearly equal one: $0.971,1.005$, and 1.024 for 4,6 , and $8 \mathrm{~m} \mathrm{~s}^{-1}$, respectively. Using a leastsquares fit to determine a linear fit of the data as:

$\frac{\varepsilon_{P L}}{\varepsilon_{a}}=m \frac{x}{M_{U}}+b$

determines the slope, $m$, of $\varepsilon_{P L} / \varepsilon_{a}$ (Table 6). Additionally, Table 6 shows the standard deviation $(\sigma)$ of the experimental data minus the value predicted by the linear fit.

Unlike the results of a zero virtual origin which showed a clear negative trend, the nonzero virtual origin results in the data scattering about the ensemble average of $0.983,1.016$, and 1.036 for 4,6 , and $8 \mathrm{~m} \mathrm{~s}^{-1}$, respectively (Fig. 15). The data scatters within $\pm 5.6 \%$ of the ensemble average for the three nominal velocities. Table 6 shows the slope $(m)$ of a linear fit to the data and the standard deviation $(\sigma)$ of the data minus the fit.

When comparing the slopes of the linear fits for a zero and a nonzero virtual origin, the assumption of a zero virtual 
origin leads to a slope at least 8 times larger than the corresponding slope for a nonzero virtual origin. The linear fits for a zero and a nonzero virtual origin produce similar $\sigma$ values at a given mean velocity, indicating that either fit represents the data. However, the power law should produce a dissipation rate equal to the dissipation rate from analog differentiation, regardless of downstream location. Although scatting about the ensemble average, only the power law with a nonzero virtual origin produces a nearly constant ratio of the dissipation rate. Therefore, self-consistency only occurs with the determination of the proper virtual origin.

\section{Conclusion}

This work presented a comparison of the dissipation rate determined from four different methods, with the analog differentiation method used as a basis of comparison. The analysis relied on hot-wire data taken downstream of an active-grid in a flow with nominal mean velocities of 4,6 , and $8 \mathrm{~m} \mathrm{~s}^{-1}$. The first method utilizes a ninth-order centraldifference scheme to numerically differentiate the temporal velocity signal. The resulting dissipation rate falls within $\pm 4 \%$ of the value determined from the analog differentiation. The second method relies on integrating the velocity power spectra to determine the dissipation rate. The analysis showed the method has a high sensitivity to noise in the signal and produces a dissipation rate 14-45\% larger than the value determined from analog differentiation. The final method relies on a power law of the dissipation rate determined by Koster (2018). The analysis shows that while a zero virtual origin and an optimized virtual origin produce qualitatively similar results, a zero virtual origin does not produce a dissipation rate consistent with the value determined from analog differentiation; specifically, the resulting dissipation rate had a negative trend with downstream location. An optimized virtual origin produces a nearly constant ratio of the dissipation rates with values of $0.983,1.016$, and 1.036 based on the ensemble average of downstream locations for 4,6 , and $8 \mathrm{~m} \mathrm{~s}^{-1}$, respectively. The data scatters within $\pm 5.6 \%$ of the ensemble average.

Open Access This article is licensed under a Creative Commons Attribution 4.0 International License, which permits use, sharing, adaptation, distribution and reproduction in any medium or format, as long as you give appropriate credit to the original author(s) and the source, provide a link to the Creative Commons licence, and indicate if changes were made. The images or other third party material in this article are included in the article's Creative Commons licence, unless indicated otherwise in a credit line to the material. If material is not included in the article's Creative Commons licence and your intended use is not permitted by statutory regulation or exceeds the permitted use, you will need to obtain permission directly from the copyright holder. To view a copy of this licence, visit http://creativecommons.org/licenses/by/4.0/.

\section{References}

Antonia R (2003) On estimating mean and instantaneous turbulent energy dissipation rates with hot wires. Exp Thermal Fluid Sci 27(2):151-157. https://doi.org/10.1016/S0894-1777(02)00259-5

Antonia RA, Smalley R, Zhou T, Anselmet F, Danaila L (2003) Similarity of energy structure functions in decaying homogeneous isotropic turbulence. J Fluid Mech 487:245-269

Champagne FH (1978) The fine-scale structure of the turbulent velocity field. J Fluid Mech 86(1):67-108

Comte-Bellot G, Corrsin S (1966) The use of a contraction to improve the isotropy of grid-generated turbulence. J Fluid Mech 25(4):657-682

del Álamo JC, Jiménez J (2009) Estimation of turbulent convection velocities and corrections to Taylor's approximation. J Fluid Mech 640:5-26. https://doi.org/10.1017/S0022112009991029

Hearst RJ, Buxton ORH, Ganapathisubramani B, Lavoie P (2012) Experimental estimation of fluctuating velocity and scalar gradients in turbulence. Exp Fluids 53(4):925-942. https://doi.org/10. 1007/s00348-012-1318-0

Heskestad G (1965) A generalized Taylor hypothesis with application for high Reynolds number turbulent shear flows. J Appl Mech 32(4):735-739

Kamruzzaman M, Djenidi L, Antonia R (2014) Effects of low Reynolds number on decay exponent in grid turbulence. Procedia Eng 90:327-332

Kang H, Chester S, Meneveau C (2003) Decaying turbulence in an active-grid-generated flow and comparisons with large-eddy simulation. J Fluid Mech 480:129-160

Kitamura T, Nagata K, Sakai Y, Sasoh A, Terashima O, Saito H, Harasaki T (2014) On invariants in grid turbulence at moderate Reynolds numbers. J Fluid Mech 738:378

Kolmogorov AN (1941) The local structure of turbulence in incompressible viscous fluid for very large Reynolds numbers. C R Acad Sci URSS 30:301-305

Koster TW (2018) Power decay law in moderate intensity nearly homogeneous isotropic turbulence. Ph.D. thesis, UC Irvine

Krogstad PÅ, Davidson P (2010) Is grid turbulence Saffman turbulence? J Fluid Mech 642:373

Lavoie P, Burattini P, Djenidi L, Antonia RA (2005) Effect of initial conditions on decaying grid turbulence at low $\mathrm{r} \lambda$. Exp Fluids 39(5):865-874

Makita H, Sassa K (1991) Active turbulence generation in a laboratory wind tunnel. In: Johansson AV, Alfredsson PH (eds) Advances in turbulence 3. Springer, Berlin, pp 497-505

Mi J, Xu M, Du C (2011) Digital filter for hot-wire measurements of small-scale turbulence properties. Meas Sci Technol 22(12):125401. https://doi.org/10.1088/0957-0233/22/12/125401

Mohamed MS, LaRue JC (1990) The decay power law in grid-generated turbulence. J Fluid Mech 219:195-214

Moin P (2010) Fundamentals of engineering numerical analysis. Cambridge University Press, Cambridge

Mordant N (2008) Experimental high Reynolds number turbulence with an active grid. Am J Phys 76(12):1092-1098

Mydlarski L, Warhaft Z (1996) On the onset of high-Reynolds-number grid-generated wind tunnel turbulence. J Fluid Mech 320:331368. https://doi.org/10.1017/S0022112096007562

Pope SB (2000) Turbulent flows. Cambridge University Press, Cambridge

Puga AJ, LaRue JC (2017) Normalized dissipation rate in a moderate Taylor Reynolds number flow. J Fluid Mech. https://doi.org/10. 1017/jfm.2017.47

Selzer O (2001) The effect of initial scalar size on the mixing of a passive scalar in grid-generated turbulence. University of California, Irvine 
Tennekes H, Lumley JL (1972) A first course in turbulence. MIT Press, Cambridge

Valente P, Vassilicos J (2011) Comment on "dissipation and decay of fractal-generated turbulence" [phys. fluids 19, 105108 (2007)]. Phys Fluids 23(11):105108.

Warhaft Z, Lumley J (1978) An experimental study of the decay of temperature fluctuations in grid-generated turbulence. J Fluid Mech 88(4):659-684
Wyngaard J (1968) Measurement of small-scale turbulence structure with hot wires. J Phys E Sci Instrum 1(11):1105

Publisher's Note Springer Nature remains neutral with regard to jurisdictional claims in published maps and institutional affiliations. 